\title{
Observer design for networked control systems implemented over WirelessHART
}

\author{
Alejandro I. Maass, Dragan Nešić, Romain Postoyan, Peter M. Dower
}

\begin{abstract}
We study the design of state observers for nonlinear networked control systems (NCSs) that are implemented over WirelessHART (WH). WH is a wireless communication protocol for process automation applications. It is characterised by its multi-hop structure, slotted communication cycles, and simultaneous transmission over different frequencies. We present a solution based on the emulation approach. That is, given an observer designed with a specific stability property in the absence of communication constraints, we implement it over a WH network and we provide sufficient conditions on the latter, to preserve the stability property of the observer. In particular, we provide explicit bounds on the maximum allowable transmission interval. We assume that the plant dynamics and measurements are affected by noise and we guarantee an inputto-state stability property for the corresponding estimation error system.
\end{abstract}

\section{INTRODUCTION}

Networked control systems (NCSs) have received much attention in the recent literature for their practical importance. Although these systems are of great significance, lots of theoretical challenges in their analysis and design remain [1], [2]. We focus on NCSs that are implemented over WirelessHART (WH), a recent wireless network used in process automation. WH is a mesh network, which utilises field devices in a multi-hop fashion. Such devices act as buffers to forward data packets. In addition, communications are precisely scheduled using time division multiple access (TDMA), and the 15 available frequency channels may be simultaneously used to transmit. Our objective is to design observers for non-linear NCSs over WH. This study is motivated by the fact that this communication protocol is increasingly used in industrial control, and that current methods for estimation over such networks [3] are obtained under strong assumptions that may be hard to implement in real WH networks (e.g. linear and discrete-time plant/observer, and equidistant transmissions). To the best of our knowledge, existing results concerning WH include optimal link scheduling [4], [5], controllercommunications co-design [6], [7], modelling and analysis [8], [9], stabilisation [10], [11], and estimation [3].

We consider the scenario where plant input and measurements are sent to an observer via a WH network. Under this setup, the observer only has access to sampled information of the plant input and output. The solution we propose

This work was supported by the ARC Discovery Scheme, grant number DP170104099, and by the ANR project COMPACS, ANR-13-BS03-004-02.

A.I. Maass, D. Nešić and P.M. Dower are with the Department of Electrical and Electronic Engineering, The University of Melbourne, Parkville, 3010, Victoria, Australia. amaass a student.unimelb.edu.au, dnesicaunimelb.edu.au, pdoweraunimelb.edu.au.

R. Postoyan is with the Université de Lorraine, CNRS, CRAN, F-54000 Nancy, France. romain.postoyanduniv-lorraine. fr. is based on emulation. The idea is to first synthesize the observer while ignoring the network. At this stage, any of the continuous-time observer design techniques available in the literature can be applied. Then, the observer is implemented over the network and conditions on the latter are derived to preserve the desired error convergence properties. Similar results on observer design exist for standard NCSs [12], [13]. However, these results are derived for abstract network models that do not correspond to any specific physical network. The recent work [14] goes one step further and solves the observer design problem for a specific physical network used in automotive control called FlexRay. However, the aforementioned results cannot be applied to our problem because of the specific properties of WH, i.e. TDMA communications, multi-hop structure and multiple frequency channels. These features require a new model, appropriate assumptions on the observer and the network, and a new stability analysis. Hence, as a first contribution, we present an observer design strategy for NCSs over WH that can cope with such features.

We model the overall NCS as a hybrid system, in the formalism of [15], that captures inter-sampling behaviour, time-varying transmissions, field device dynamics, and nonlinear plant and observer. In [11], the same formalism was used to obtain a model of an NCS over WH in the context of stabilisation. Under the observer design setup, and as our second contribution, we consider plants with perturbed dynamics and noisy measurements, which are more general and induce additional technical difficulties compared with [11]. We assume that transmissions over the network are governed by uniformly globally exponentially stable (UGES) protocols, which is a property satisfied by many protocols implementable in WH [11]. As our third and final contribution, we provide explicit bounds on the maximum allowable transmission interval (MATI) that guarantee an input-to-state (ISS) stability property for the estimation error system.

Notation: Denote by $\mathbb{R}$ the set of real numbers, $\mathbb{R}^{n}$ the set of all real vectors with $n$ components, $\mathbb{R}_{\geq 0} \doteq[0, \infty)$, $\mathbb{R}_{>0} \doteq(0, \infty), \mathbb{Z}_{\geq 0} \doteq\{0,1,2, \ldots\}$ and $\mathbb{N} \doteq\{1,2, \ldots\}$. For vector arguments, $|\cdot|$ denotes the Euclidean norm. For simplicity, we use $(x, y) \doteq\left[x^{T} y^{T}\right]^{T} \in \mathbb{R}^{n+m}$, for all $x \in$ $\mathbb{R}^{n}$ and $y \in \mathbb{R}^{m}$. A function $\alpha: \mathbb{R}_{>0} \rightarrow \mathbb{R}_{>0}$ is of class $\mathcal{K}$ if it is continuous, zero at zero and strictly increasing. It is of class $\mathcal{K}_{\infty}$ if it is of class $\mathcal{K}$ and unbounded. A function $\eta: \mathbb{R}_{\geq 0} \times \mathbb{R}_{\geq 0} \rightarrow \mathbb{R}_{\geq 0}$ is of class $\mathcal{K} \mathcal{K}$ if $\eta\left(s_{1}, \cdot\right)$ and $\eta\left(\cdot, s_{2}\right)$ are of class $\mathcal{K}$ for each $s_{1} \geq 0$ and $s_{2} \geq 0$. A function $\beta: \mathbb{R}_{\geq 0} \times \mathbb{R}_{\geq 0} \rightarrow \mathbb{R}_{\geq 0}$ is of class $\mathcal{K} \mathcal{L}$ if $\beta(\cdot, t)$ is of class $\mathcal{K}$ for each $t \geq 0$, and if $\beta(s, \cdot)$ is continuous, non-increasing 


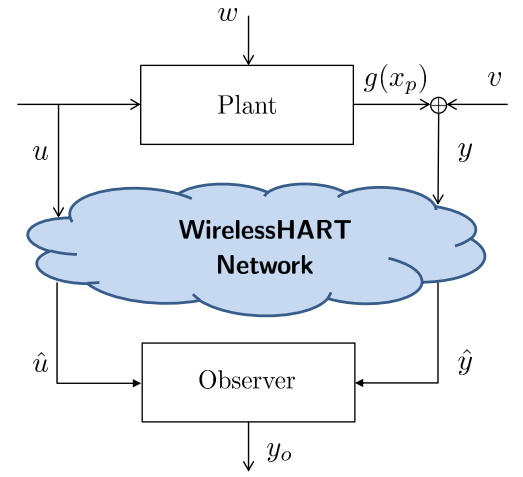

Fig. 1. Block diagram of our networked observer architecture.

and satisfies $\lim _{t \rightarrow \infty} \beta(s, t)=0$ for each $s \geq 0$. Given $t \in \mathbb{R}$ and a piecewise continuous function $f: \mathbb{R} \rightarrow \mathbb{R}^{n}$, we use the notation $f\left(t^{+}\right) \doteq \lim _{s \rightarrow t, s>t} f(s)$. We use $\mathbf{1}_{n}$ to denote $(1, \ldots, 1)^{T} \in \mathbb{R}^{n}$, and $\mathbb{1}_{S}$ as the function $\mathbb{1}_{S}: \mathbb{N} \rightarrow\{0,1\}$ such that $\mathbb{1}_{S}(i)=1$ if $i \in S$, and $\mathbb{1}_{S}(i)=0$ if $i \notin S$.

\section{SetUP AND PROBLEM DEFINITION}

Consider a non-linear plant

$$
\dot{x}_{p}=f\left(x_{p}, u, w\right), \quad y=g\left(x_{p}\right)+v,
$$

where $x_{p} \in \mathbb{R}^{n_{x}}$ is the state, $u \in \mathbb{R}^{n_{u}}$ is the control input, $w \in \mathbb{R}^{n_{w}}$ is the external disturbance, $y \in \mathbb{R}^{n_{y}}$ is the plant output affected by the noise $v \in \mathbb{R}^{n_{y}}$, and $n_{x}, n_{u}, n_{w}, n_{y} \in \mathbb{N}$. The functions $u: \mathbb{R} \rightarrow \mathbb{R}^{n_{u}}$ and $v: \mathbb{R} \rightarrow \mathbb{R}^{n_{v}}$ are assumed to be Lebesgue measurable and differentiable almost everywhere. Moreover, these functions and their time-derivatives are assumed to have a finite $\mathcal{L}_{\infty}$ norm. We assume an observer has been designed for the above plant and has the form

$$
\dot{x}_{o}=f_{o}\left(x_{o}, u, y-y_{o}\right), \quad y_{o}=g\left(x_{o}\right),
$$

where $x_{o} \in \mathbb{R}^{n_{x}}$ is the estimate of the state $x_{p}$, and $y_{o} \in \mathbb{R}^{n_{y}}$ is the output estimate. It is implicit in (2) that we measure $u$ and $y$, whereas $w$ and $v$ are unmeasured. The stability property we will prove is natural for this setting.

We study the scenario depicted in Fig. 1, in which the plant communicates with the observer over a WH network. In particular, the control signal $u$ is directly available to the plant and transmitted to the observer via the network. We denote the control signal received by the observer by $\hat{u}$. The noisy output $y$ is also transmitted to the observer via the network. The output received by the observer is likewise denoted by $\hat{y}$. We emphasize that the observer has no longer access to $(u, y)$ as in classical control theory, but to their networked versions $(\hat{u}, \hat{y})$.

Under the setup described above, our main objective is to provide conditions on the observer and the network, in particular on the scheduling protocols and the MATI, under which the state of the observer (approximately) converges to the state of the plant.

\section{WIRELESSHART NETWORK}

We briefly present a description of WH (see, e.g. [16]) and the adopted assumptions.

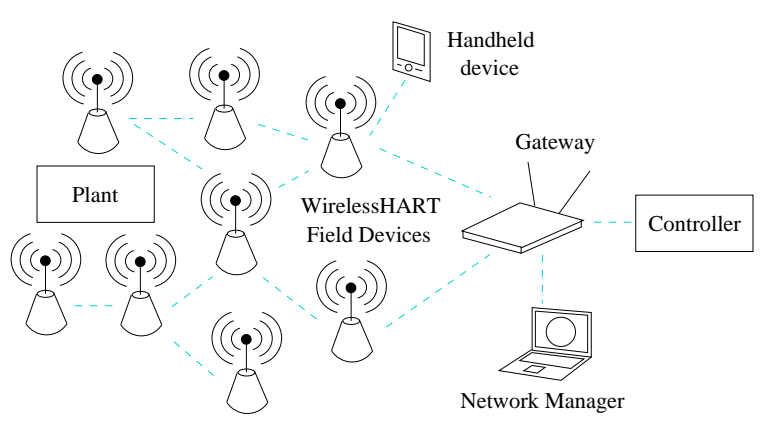

Fig. 2. WirelessHART architecture.

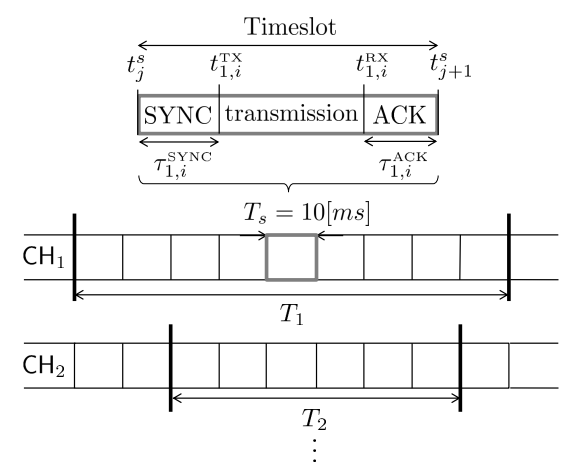

Fig. 3. WirelessHART superframe table.

\section{A. Communication features}

The general architecture of a WH network is shown in Fig. 2. It consists of an interconnection of: field devices that communicate with the plant process (e.g. sensor/actuators), handheld devices to run diagnostics, gateways that enable communications between host applications and field devices, and a network manager responsible of the scheduling between devices. WH is based on the IEEE 802.15.4-2006 physical layer and operates in the $2.4 \mathrm{GHz}$ ISM radio band over 15 frequency division multiplexed channels. In the data link layer, WH defines a slotted TDMA technology. WH networks also support multiple access timeslots, where multiple devices can share a specific channel, but these are out of the scope of this paper.

\section{B. TDMA superframe structure}

All communications in a WH network are defined with respect to a superframe. For each $l$-th channel, $l=1, \ldots, 15$, a superframe is an a priori fixed period of time $T_{l}>0$, contiguous in real time with other superframes within each channel, that is divided into a sequence of timeslots as depicted in Fig. 3. Field devices are scheduled to transmit in the superframe, and each one of the 15 channels may have a different superframe depending on the chosen scheduling protocol. The set of superframes across frequency channels is called a superframe table. Each timeslot is strictly $T_{s}=10[\mathrm{~ms}]$ in duration. Within this timeslot, a complete single data packet and its corresponding acknowledgement are transmitted between two field devices. The transmission delay required for the delivery of a packet from a transmitting device to a receiving device, in each $l$-th channel and $i$-th timeslot, is denoted by $t_{l, i}^{\mathrm{RX}}-t_{l, i}^{\mathrm{TX}}$ and it depends on the 
packet size. For each channel $l$-th channel and at the end of every $i$-th timeslot, the time it takes to acknowledge such a packet is denoted by $\tau_{l, i}^{\text {ACK }}$. In order to have effective TDMA communications, all devices need to be synchronized. This is ensured, at the beginning of each $i$-th timeslot on the $l$-th channel, via the introduction of a synchronising variable delay of duration $\tau_{l, i}^{\mathrm{SYNC}}$. For simplicity, and similar to [10], [11], the following additional assumption is adopted.

Assumption 1: The following holds.

(a) Transmissions across all channels are synchronised, i.e. $\tau_{i}^{\mathrm{SYNC}} \doteq \tau_{l, i}^{\mathrm{SYNC}}=\tau_{k, i}^{\mathrm{SYNC}}$ for all $l, k \in\{1, \ldots, 15\}$ and $i \in \mathbb{N}$.

(b) Acknowledgement time is negligible in each timeslot, i.e. $\tau_{l, i}^{\mathrm{ACK}}=0$ for all $l \in\{1, \ldots, 15\}$ and $i \in \mathbb{N}$.

(c) Packets are transmitted instantaneously in every timeslot, i.e. $t_{i} \doteq t_{l, i}^{\mathrm{TX}}=t_{l, i}^{\mathrm{RX}}$ for all $l \in\{1, \ldots, 15\}$ and $i \in \mathbb{N}$. (We refer to $t_{i}$ as transmission instant.)

(d) One successful transmission between devices occurs within each timeslot, per channel.

(e) The transmission instants satisfy $\varepsilon \leq t_{i+1}-t_{i} \leq \tau_{\text {MATI }} \leq$ $2 T_{s}-\varepsilon$ for all $i \in \mathbb{N}$, where $0<\varepsilon \doteq \inf _{i \in \mathbb{N}} \tau_{i}^{\text {SYNC }}$.

In Assumption 1, $\tau_{\text {MATI }}$ corresponds to the so-called maximal allowable transmission interval (MATI) [17]. We now introduce two clock variables which are useful to model the NCS in Fig. 1, in the hybrid formalism of [15]. We use $\tau \in \mathbb{R}_{\geq 0}$ to represent the time elapsed since the last transmission. We need an additional clock variable $\tau_{s} \in \mathbb{R}_{\geq 0}$, which corresponds to the time elapsed on each timeslot. Also, let us introduce a counter $q \in\{0,1\}$ to limit transmissions within the timeslot to one. We can model the above variables to satisfy Assumption 1 as

$$
\left.\begin{array}{rl}
\dot{\tau} & =1 \\
\dot{\tau}_{s} & =1 \\
\dot{q} & =0
\end{array}\right\}\left(\tau, \tau_{s}, q\right) \in\left[0, \tau_{\mathrm{MATI}}\right] \times\left[0, T_{s}\right] \times\{0,1\},
$$

The above equations model the fact that there is only one transmission within a timeslot on a given frequency, and the fact that a packet must be transmitted at most in $\tau_{\text {MATI }}$ seconds.

\section{OBSERVER EMULATION}

We implement the observer (2) in the setup of Fig. 1, i.e.

$$
\dot{x}_{o}=f_{o}\left(x_{o}, \hat{u}, \hat{y}-\hat{y}_{o}\right),
$$

where we note that the emulated observer (4) no longer depends on $(y, u)$, but on $(\hat{y}, \hat{u})$ because of the network. Furthermore, we note that (4) does not depend on its output $y_{o}$, as in (2), but on $\hat{y}_{o}$, which is a networked version of $y_{o}$. The idea of using $\hat{y}_{o}$ instead of $y_{o}$ was suggested in [13, Section VIII] and allows stronger stability properties for the

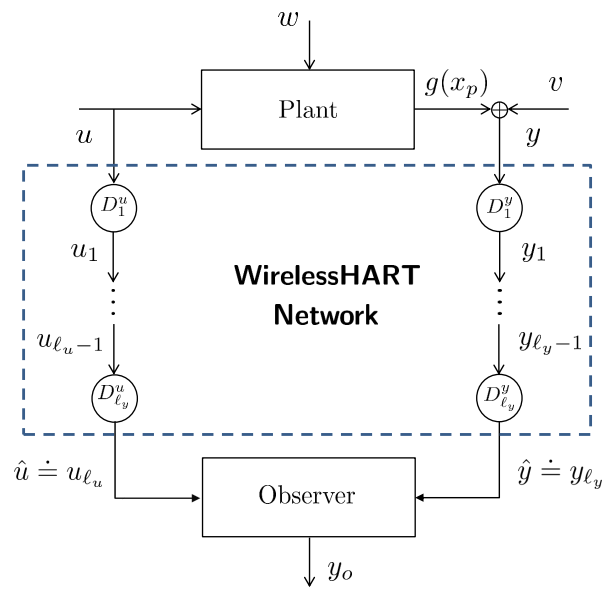

Fig. 4. NCS implemented over a WH network with $\ell_{y}$ field devices in the $y$-path and $\ell_{u}$ field devices in the $u$-path.

estimation error system to be established. A similar idea is proposed in [18] for the design of high-gain observers.

We next explain how $\hat{u}, \hat{y}$ and $\hat{y}_{o}$ behave given the $\mathrm{WH}$ network. Consider Fig. 4, in which the WH network contains $\ell_{y} \in \mathbb{Z}_{\geq 0}$ field devices interconnected in the $y$-path and $\ell_{u} \in \mathbb{Z}_{>0}$ in the $u$-path. We label the field devices as $D_{\alpha}^{y}$ and $D_{\beta}^{u}$, where $\alpha=1, \ldots, \ell_{y}$ and $\beta=1, \ldots, \ell_{u}$. For each field device, its inputs and outputs are depicted in Fig. 4. Note that the signals that actually reach the observer in Fig. 4, i.e. $y_{\ell_{y}}$ and $u_{\ell_{u}}$, are denoted as $\hat{y}$ and $\hat{u}$, to be consistent with Fig. 1 and existent NCS literature. We model field devices as buffers, for which we introduce a buffer state variable, denoted by $b_{\alpha}^{y}$ and $b_{\beta}^{u}$ for field devices in the $y$-path and $u$ path, respectively. In the following, we explain the reception and transmission behaviour of field devices, and we present the equations associated with this process.

Reception: Suppose a field device $D_{\alpha}^{y}$ receives a packet at time instant $t_{i}$. Then, $D_{\alpha}^{y}$ updates the content of its buffer via its input. During this process, the output of $D_{\alpha}^{y}$ remains unchanged. We write this as follows,

$$
\begin{aligned}
\dot{y}_{\alpha}(t) & =0, \quad t \in\left[t_{i}, t_{i+1}\right], \\
\dot{b}_{\alpha}^{y}(t) & =0, \quad t \in\left[t_{i}, t_{i+1}\right], \\
b_{\alpha}^{y}\left(t_{i}^{+}\right) & =y_{\alpha-1}\left(t_{i}^{+}\right), \\
y_{\alpha}\left(t_{i}^{+}\right) & =y_{\alpha}\left(t_{i}\right),
\end{aligned}
$$

for all $\alpha=1, \ldots, \ell_{y}$. Note that $y_{0} \equiv y$ for $\alpha=1$, i.e. device one samples the value of the plant output.

Transmission: Suppose a field device $D_{\alpha}^{y}$ is scheduled to transmit at time instant $t_{i}$. Here, $D_{\alpha}^{y}$ sends the content of its buffer through its output, and keeps it until a new packet is received. This can be written as follows,

$$
\begin{aligned}
\dot{y}_{\alpha}(t) & =0, \quad t \in\left[t_{i}, t_{i+1}\right], \\
\dot{b}_{\alpha}^{y}(t) & =0, \quad t \in\left[t_{i}, t_{i+1}\right], \\
y_{\alpha}\left(t_{i}^{+}\right) & =b_{\alpha}^{y}\left(t_{i}\right), \\
b_{\alpha}^{y}\left(t_{i}^{+}\right) & =b_{\alpha}^{y}\left(t_{i}\right),
\end{aligned}
$$

for all $\alpha=1, \ldots, \ell_{y}$.

We introduce the network-induced errors of the $y$-path and $u$-path, which we denote by $\zeta^{y} \in \mathbb{R}^{n_{\zeta^{y}}}, n_{\zeta^{y}} \doteq 2 \ell_{y} n_{y}$, and 
$\zeta^{u} \in \mathbb{R}^{n_{\zeta^{u}}}, n_{\zeta^{u}} \doteq 2 \ell_{u} n_{u}$, respectively. That is,

$$
\begin{aligned}
\zeta^{\star} \doteq\left(b_{1}^{\star}-\star, b_{2}^{\star}-\star_{1}, \ldots, b_{\ell_{\star}}^{\star}-\star_{\ell_{\star}-1},\right. \\
\left.\star_{1}-b_{1}^{\star}, \star_{2}-b_{2}^{\star}, \ldots, \star_{\ell_{\star}}-b_{\ell_{\star}}^{\star}\right),
\end{aligned}
$$

for $\star \in\{y, u\}$. The first $\ell_{\star}$ components of $\zeta^{\star}$ are related to the buffer update during reception. The remaining $\ell_{\star}$ components of $\zeta^{\star}$ are related to the transmission of such buffer value through their output. In particular, we will reset to zero these errors to model reception and transmission. This is a major difference with previous models of non-linear NCSs treated in [17], [19], [20], where the network-induced error is given by $e \doteq(\hat{y}-y, \hat{u}-u)$ (i.e. no specific network is considered, and buffer dynamics are ignored).

The artificial variable $\hat{y}_{o}$ is the "networked version" of $y_{o}$. It evolves along the same vector field as $\hat{y}$ between two successive transmission instants, i.e. $\dot{\hat{y}}_{o}=0$ for $t \in$ $\left[t_{i}, t_{i+1}\right]$. At jumps, it evolves exactly as when $y$ is sent through the network. Therefore, for analysis purposes, we introduce auxiliary field devices with buffer states $b_{1}^{o}, \ldots, b_{\ell_{y}}^{o}$ and outputs $y_{o, 1}, \ldots, \hat{y}_{o}$. Then, we define the corresponding network induced error on the observer output

$$
\begin{aligned}
\zeta^{y_{o}} \doteq\left(b_{1}^{o}-y_{o}, b_{2}^{o}-y_{o, 1}, \ldots, b_{\ell_{y}}^{o}-y_{o, \ell_{y}-1},\right. \\
\left.y_{o, 1}-b_{1}^{o}, y_{o, 2}-b_{2}^{o}, \ldots, \hat{y}_{o}-b_{\ell_{y}}^{o}\right) .
\end{aligned}
$$

This will help to ensure that the update of $\hat{y}_{o}$ with $y_{o}$ happens at the same time that $\hat{y}$ gets updated with $y$, so they can be properly compared through the observation error $y-y_{o}$. Hence, we artificially send $y_{o}$ through a model of the $y$-path of the network at the same time that $y$ is sent, to construct $\hat{y}_{o}$. The introduction of $\zeta^{y_{o}}$ is a key difference with previous work on observer design [12]-[14], and it is used here as a consequence of how WH operates.

Using the definitions of $\zeta^{y}, \zeta^{u}$ and $\zeta^{y_{o}}$ in (4), we get

$$
\dot{x}_{o}=f_{o}\left(x_{o}, u+\mathbf{1}_{2 \ell_{u}} \cdot \zeta^{u}, y-y_{o}+\mathbf{1}_{2 \ell_{y}} \cdot \zeta^{e}\right),
$$

where $\zeta^{e} \doteq \zeta^{y}-\zeta^{y_{o}}$ corresponds to the network induced error on the observation error $y-y_{o}$. The network-induced errors in (7) and (8) are useful to model scheduling in WH networks. Given that the observer dynamics in (9) are affected by $\zeta^{e}$ and $\zeta^{u}$, we define $\varsigma \doteq\left(\zeta^{e}, \zeta^{u}\right)$, where $\varsigma \in \mathbb{R}^{n_{\varsigma}}, n_{\varsigma} \doteq n_{\zeta^{y}}+n_{\zeta^{u}}$.

\section{SCHEDULING PROTOCOLS}

We introduce the protocol equation of $\zeta^{y}$ and $\zeta^{u}$, that is $\zeta^{y}\left(t_{i}^{+}\right)=\mathcal{H}^{y}(i) \zeta^{y}\left(t_{i}\right)$ and $\zeta^{u}\left(t_{i}^{+}\right)=\mathcal{H}^{u}(i) \zeta^{u}\left(t_{i}\right)$, respectively. The time-varying matrices $\mathcal{H}^{y}(i)$ and $\mathcal{H}^{u}(i)$, for all $i \in \mathbb{N}$, are matrices containing only ones and zeros so that the corresponding error component is reset to zero according to the implemented scheduling protocol. Because of the way $\hat{y}_{o}$ is constructed, its protocol equation is exactly as the one of $\zeta^{y}$, that is, $\zeta^{y_{o}}\left(t_{i}^{+}\right)=\mathcal{H}^{y}(i) \zeta^{y_{o}}\left(t_{i}\right)$. Therefore, for $\zeta^{e}$ we have that $\zeta^{e}\left(t_{i}^{+}\right)=\mathcal{H}^{y}(i) \zeta^{e}\left(t_{i}\right)$. Then, the corresponding protocol equation for the network-induced error $\varsigma$ is

$$
\varsigma\left(t_{i}^{+}\right)=h_{\varsigma}\left(i, \varsigma\left(t_{i}\right)\right) \doteq \operatorname{diag}\left\{\mathcal{H}^{y}(i), \mathcal{H}^{u}(i)\right\} \varsigma\left(t_{i}\right) .
$$

TABLE I

SUPERFRAME TABLE FOR THE FDD-RR PROTOCOL.

\begin{tabular}{c|c|c|c|c|c|} 
& $t_{1}$ & $t_{2}$ & $\cdots$ & $t_{\ell_{y}}$ & $t_{\ell_{y}+1}$ \\
\hline $\mathrm{CH}_{1}$ & $P \rightarrow D_{1}^{y}$ & $D_{1}^{y} \rightarrow D_{2}^{y}$ & $\cdots$ & $D_{\ell_{y-1}}^{y} \rightarrow D_{\ell_{y}}^{y}$ & $D_{\ell_{y}}^{y} \rightarrow C$ \\
\hline $\mathrm{CH}_{2}$ & $C \rightarrow D_{1}^{u}$ & $D_{1}^{u} \rightarrow D_{2}^{u}$ & $\cdots$ & $D_{\ell_{u}}^{u} \rightarrow P$ & \\
\hline
\end{tabular}

We assume that the scheduling rule (10) satisfies the following properties.

Assumption 2: There exist $W: \mathbb{Z}_{\geq 0} \times \mathbb{R}^{n_{\varsigma}} \rightarrow \mathbb{R}_{\geq 0}$ that is locally Lipschitz in its second argument, constants $\underline{\alpha}_{W}, \bar{\alpha}_{W}>0$, and a real number $\lambda \in(0,1)$, such that, for all $\kappa \in \mathbb{Z}_{\geq 0}$ and $\varsigma \in \mathbb{R}^{n_{\varsigma}}$,

$$
\begin{aligned}
\underline{\alpha}_{W}|\varsigma| & \leq W(\kappa, \varsigma) \leq \bar{\alpha}_{W}|\varsigma|, \\
W\left(\kappa+1, h_{\varsigma}(\kappa, \varsigma)\right) & \leq \lambda W(\kappa, \varsigma) .
\end{aligned}
$$

Assumption 2 is a stability condition on the protocol equation (10). More precisely, Assumption 2 states that (10) is a uniformly globally exponentially stable (UGES) protocol, as termed in [20]. We next provide an example of a scheduling protocol, that is implementable in $\mathrm{WH}$, and satisfies these conditions. In fact, many other WH protocols satisfy Assumption 2, see e.g. [11].

Example 1: We consider a scheduling protocol that establishes a full-duplex communication link that uses two different frequency channels for measurements and actuation operations. In particular, we consider the protocol in Table I. We call this protocol Frequency Division Duplex Round Robin (FDD-RR). For this protocol, we have that $\mathcal{H}^{\star}$ in $(10), \star \in\{y, u\}$, is given by

$$
\begin{aligned}
\mathcal{H}^{\star}(i) & \doteq\left[\begin{array}{cc}
\Delta^{\star}(i) & 0 \\
I-\Delta^{\star}(i) & \Gamma^{\star}(i)
\end{array}\right], \\
\Delta^{\star}(i) & \doteq \operatorname{diag}\left\{\delta_{1}^{\star}(i) I_{n_{\star}}, \ldots, \delta_{\ell_{\star}}^{\star}(i) I_{n_{\star}}\right\}, \\
\Gamma^{\star}(i) & \doteq\left[\begin{array}{cc}
\gamma_{1}^{\star}(i) I_{n_{\star}} & 0 \\
\left(1-\gamma_{1}^{\star}(i)\right) I_{n_{\star}} \gamma_{2}^{\star}(i) I_{n_{\star}} & \\
\ddots & \ddots \\
0 & \left(1-\gamma_{\ell_{\star}-1}^{\star}(i)\right) I_{n_{\star}} \gamma_{\ell_{\star}}(i) I_{n_{\star}}
\end{array}\right],
\end{aligned}
$$

with $\delta_{\alpha}^{y}(i) \doteq 1-\mathbb{1}_{\mathcal{D}_{\alpha}^{y}}(i), \delta_{\beta}^{u}(i) \doteq 1-\mathbb{1}_{\mathcal{D}_{\beta}^{u}}(i), \gamma_{\alpha}^{y}(i) \doteq$ $1-\mathbb{1}_{\overline{\mathcal{D}}_{\alpha}^{y}}(i)$, and $\gamma_{\beta}^{u}(i) \doteq 1-\mathbb{1}_{\overline{\mathcal{D}}_{\beta}^{u}}(i)$, where $\mathcal{D}_{\alpha}^{y} \doteq\{i \in \mathbb{N}$ : $\left.i=\alpha+\left(\ell_{y}+1\right) \sigma, \sigma \in \mathbb{Z}_{\geq 0}\right\}, \mathcal{D}_{\beta}^{u} \doteq\left\{i \in \mathbb{N}: i=\beta+\left(\ell_{u}+\right.\right.$ 1) $\left.\sigma, \sigma \in \mathbb{Z}_{\geq 0}\right\}, \overline{\mathcal{D}}_{\alpha}^{y} \doteq\left\{i \in \mathbb{N}: i=\alpha+1+\left(\ell_{y}+1\right) \sigma, \sigma \in\right.$ $\left.\mathbb{Z}_{\geq 0}\right\}, \overline{\mathcal{D}}_{\beta}^{y} \stackrel{\doteq}{=}\left\{i \in \mathbb{N}: i=\beta+1+\left(\ell_{u}+1\right) \sigma, \sigma \in \mathbb{Z}_{\geq 0}\right\}$, for $\alpha=1, \ldots, \ell_{y}$ and $\beta=1, \ldots, \ell_{u}$. It is possible to show that the FDD-RR protocol satisfies Assumption 2 with $\underline{\alpha}_{W}=1$, $\bar{\alpha}_{W}=\sqrt{\frac{3^{N}-1}{2}}$ and $\lambda=\sqrt{\frac{3^{N}-3}{3^{N}-1}}, N=\max \left\{2 \ell_{y}+1,2 \ell_{u}+\right.$ $1\}$, with Lyapunov function $W(i, \varsigma) \doteq \sqrt{\sum_{k=i}^{\infty}|\phi(k, i, \varsigma)|^{2}}$, in which $\phi(k, i, \varsigma)$ corresponds to the solution of $\varsigma(i+1)=$ $\mathcal{H}(i) \varsigma(i)$ at time $k$ starting at time $i$ and initial condition $\varsigma$.

\section{MAIn RESUlts}

In this section we first provide the hybrid model for the NCS of Fig. 4. We then proceed to use this model to obtain an explicit bound on the MATI that guarantees an ISS stability property for the estimation error system. 


\section{A. NCS model}

We introduce the variable $\kappa \in \mathbb{Z}_{>0}$, which counts the number of transmissions. We also introduce the estimation error $\chi \doteq x_{o}-x_{p}$, and $d \doteq\left(d_{u}, d_{v}\right)$, where $d_{u}$ and $d_{v}$ denote the time derivative of the input signal $u$ and of the noise $v$, respectively. Let $\psi \doteq\left(\chi, x_{p}, \varsigma, \tau, \kappa, \tau_{s}, q\right), \psi \in \mathbb{S} \doteq$ $\mathbb{R}^{2 n_{x}+n_{\varsigma}} \times \mathbb{R}_{\geq 0} \times \mathbb{Z}_{\geq 0} \times \mathbb{R}_{\geq 0} \times\{0,1\}$, be the full state vector. In view of Sections II-V, we model the overall system using the hybrid formalism of [15], which gives

$$
\begin{aligned}
\dot{\psi} & =\mathcal{F}(\psi, u, v, w, d), \quad \psi \in C, \\
\psi^{+} & =\mathcal{G}(\psi), \quad \psi \in D,
\end{aligned}
$$

where $C \doteq \mathbb{R}^{2 n_{x}+n_{\varsigma}} \times\left[0, \tau_{\text {MATI }}\right] \times \mathbb{Z}_{\geq 0} \times\left[0, T_{s}\right] \times\{0,1\}, D \doteq$ $D^{\text {trans }} \cup D^{\text {slot }}, D^{\text {trans }} \doteq \mathbb{R}^{2 n_{x}+n_{\varsigma}} \times\left[\varepsilon, \tau_{\text {MATI }}\right] \times \mathbb{Z}_{\geq 0} \times\left[\varepsilon, T_{s}\right] \times$ $\{0\}, D^{\text {slot }} \doteq \mathbb{R}^{2 n_{x}+n_{\varsigma}} \times\left[\varepsilon, \tau_{\text {MATI }}\right] \times \mathbb{Z}_{\geq 0} \times\left\{T_{s}\right\} \times\{1\}$. The mappings $\mathcal{F}$ and $\mathcal{G}$ are defined as

$$
\begin{aligned}
& \mathcal{F}(\psi, u, v, w, d) \doteq\left(f_{\chi}(\psi, u, v, w), f_{p}(\psi, u, v, w),\right. \\
&\left.g_{\varsigma}(\psi, u, v, w, d), 1,0,1,0\right), \\
& \mathcal{G}(\psi) \doteq \begin{cases}\mathcal{G}^{\text {trans }}(\psi), & \xi \in D^{\text {trans }} \\
\mathcal{G}^{\operatorname{slot}}(\psi), & \xi \in D^{\text {slot }}\end{cases}
\end{aligned}
$$

where $\mathcal{G}^{\text {trans }}(\psi) \doteq\left(\chi, x_{p}, h_{\varsigma}(\kappa, \varsigma), 0, \kappa+1, \tau_{s}, 1-q\right)$, $\mathcal{G}^{\text {slot }}(\psi) \doteq\left(\chi, x_{p}, \varsigma, \tau, \kappa, 0,0\right)$, and

$$
\begin{aligned}
& f_{\chi}(\psi, u, v, w) \doteq f_{o}\left(x_{p}+\chi, u+\mathbf{1}_{2 \ell_{u}} \cdot \zeta^{u}, \mathbf{1}_{2 \ell_{y}} \cdot \zeta^{e}\right. \\
&+\left.g\left(x_{p}\right)+v-g\left(x_{p}+\chi\right)\right)-f\left(x_{p}, u, w\right), \\
& f_{p}(\psi, u, v, w) \doteq f\left(x_{p}, u, w\right), \\
& g_{\varsigma}(\psi, u, v, w, d) \doteq\left(g_{\varsigma, 1}(\psi, u, v, w, d), 0, \ldots, 0,\right. \\
&\left.\quad-d_{u}, 0, \ldots, 0\right), \\
& g_{\varsigma, 1}(\psi, u, v, w, d) \doteq-\frac{\partial g}{\partial x_{p}} f\left(x_{p}, u, w\right)-d_{v} \frac{\partial g}{\partial x_{o}} f_{o}\left(x_{p}+\chi,\right. \\
&\left.u+\mathbf{1}_{2 \ell_{u}} \cdot \zeta^{u}, \mathbf{1}_{2 \ell_{y}} \cdot \zeta^{e}+g\left(x_{p}\right)+v-g\left(x_{p}+\chi\right)\right) .
\end{aligned}
$$

We can see that the hybrid system (12) is subject to two different jumps, namely transmission jumps (when $\psi \in$ $D^{\text {trans }}$ ) and timeslot switching jumps (when $\psi \in D^{\text {slot }}$ ).

\section{B. Stability analysis}

We first assume an exponential growth condition on the ऽ-subsystem, consistent with [12], [13].

Assumption 3: There exist a continuous function $H$ : $\mathbb{R}^{n_{x}} \rightarrow \mathbb{R}_{\geq 0}$, a function $\sigma \in \mathcal{K}_{\infty}$ and constant $L \geq 0$ such that for all $\chi \in \mathbb{R}^{n_{x}}, u \in \mathbb{R}^{n_{u}}, v \in \mathbb{R}^{n_{y}}, w \in \mathbb{R}^{n_{w}}, d \in$ $\mathbb{R}^{n_{u}+n_{y}}, \kappa \in \mathbb{Z}_{\geq 0}$ and almost all $\varsigma \in \mathbb{R}^{n_{\varsigma}}$,

$$
\begin{array}{r}
\left\langle\frac{\partial W(\kappa, \varsigma)}{\partial \varsigma}, g_{\varsigma}(\psi, u, v, w, d)\right\rangle \leq L W(\kappa, \varsigma)+H(\chi) \\
+\sigma(|(u, v, w, d)|)
\end{array}
$$

where the function $W$ comes from Assumption 2.

The above condition is satisfied when: $W$ is globally Lipschitz in $\varsigma$ with constant $S_{1} \geq 0$ (which is the case for the protocol in Example 1), and when $\left|g_{\varsigma}(\psi, u, v, w, d)\right| \leq$ $S_{2}|\varsigma|+S_{3}|\chi|+S_{4}|(u, v, w, d)|$ for all $(\psi, u, v, w, d) \in \mathbb{S} \times$ $\mathbb{R}^{2 n_{u}+2 n_{y}+n_{w}}$, with $S_{2}, S_{3}, S_{4}>0$. In this case, Assumption
3 is satisfied with $L \doteq S_{1} S_{2} / \underline{\alpha}_{W}, H(\chi) \doteq S_{1} S_{3}|\chi|$ and $\sigma(s) \doteq S_{1} S_{4} s$ for all $\chi \in \mathbb{R}^{n_{x}}$ and $s \geq 0$, where $\underline{\alpha}_{W}>0$ comes from Assumption 2.

We next assume that the observer (2) has been designed such that the following conditions hold.

Assumption 4: There exist a continuously differentiable function $V: \mathbb{R}^{n_{x}} \rightarrow \mathbb{R}_{\geq 0}$, functions $\underline{\alpha}_{V}, \bar{\alpha}_{V}, \mu \in \mathcal{K}_{\infty}$, and constants $\varrho, \gamma>0$ such that for all $\chi \in \mathbb{R}^{n_{x}}, \varsigma \in \mathbb{R}^{n_{\varsigma}}, v \in$ $\mathbb{R}^{n_{y}}, w \in \mathbb{R}^{n_{w}}$ and $\kappa \in \mathbb{Z}_{\geq 0}$,

$$
\begin{gathered}
\underline{\alpha}_{V}(|\chi|) \leq V(\chi) \leq \bar{\alpha}_{V}(|\chi|), \\
\left\langle\nabla V(\chi), f_{\chi}(\psi, u, v, w)\right\rangle \leq-\varrho V(\chi)-\varrho W(\varsigma, \kappa)^{2} \\
-H(\chi)^{2}+\gamma^{2} W(\kappa, \varsigma)^{2}+\mu(|(v, w)|),
\end{gathered}
$$

where $W$ and $H$ come from Assumptions 2 and 3, respectively.

Assumption 4 ensures that observer (2) is robust for plant (1). In particular, it implies that the $\chi$-system in (12), which describes the evolution of the estimation error, is $\mathcal{L}_{2}$ stable from $(W, \sqrt{\mu})$ to $H$, and ISS with respect to $(v, w)$.

Since all the required assumptions have been stated, we can now present the stability property of the observer.

Theorem 1: Suppose that Assumptions 2-4 hold for system (12), and that given any $\epsilon>0, \tau_{\text {MATI }}$ satisfies

$$
\tau_{\text {MATI }} \leq \begin{cases}\frac{1}{L r} \arctan \left(\frac{\lambda}{\frac{\lambda}{1+\lambda}\left(\frac{(2+\epsilon) \gamma}{L}-2\right)+1+\lambda}\right), & \gamma>\frac{L}{\sqrt{1+\epsilon}}, \\ \frac{1}{L \sqrt{1+\epsilon}} \frac{1-\lambda}{1+\lambda}, & \gamma=\frac{L}{\sqrt{1+\epsilon}} \\ \frac{1}{L r} \operatorname{arctanh}\left(\frac{(1-\lambda) r}{\frac{\lambda}{1+\lambda}\left(\frac{(2+\epsilon) \gamma}{L}-2\right)+1+\lambda}\right), & \gamma<\frac{L}{\sqrt{1+\epsilon}}\end{cases}
$$

where $r \doteq \sqrt{\left|\gamma^{2}(1+\epsilon) / L^{2}-1\right|}$, and $\lambda, L$ and $\gamma$ are as per Assumptions 2, 3 and 4, respectively. Then there exist $\beta \in \mathcal{K} \mathcal{L}, \eta_{1} \in \mathcal{K}_{\infty}$, and $\eta_{2} \in \mathcal{K} \mathcal{K}$ such that each solution pair $^{1}(\psi,(u, v, w, d))$ to system (12) satisfies

$$
\begin{array}{r}
|\psi(t, j)|_{\mathcal{A}} \leq \beta\left(|\psi(0,0)|_{\mathcal{A}}, t+j\right)+\eta_{1}\left(\|(v, w)\|_{(t, j)}\right) \\
+\eta_{2}\left(1 / \epsilon,\|(u, v, w, d)\|_{(t, j)}\right),
\end{array}
$$

for all $(t, j) \in \operatorname{dom} \psi$, where $\mathcal{A} \doteq\{\psi \in \mathbb{S}: \chi=0, \varsigma=0\}$. We can see from Theorem 1 that the estimation error $\chi$ and the network-induced error $\varsigma$ converge to a ball centred at the origin and whose radius depends on the $\mathcal{L}_{\infty}$ norm of the input $(u, v, w, d)$. We can see that $\chi$ and $\varsigma$ do not a priori converge to the origin even when $w=v=0$, since in this case we have that $|\psi(t, j)|_{\mathcal{A}} \leq \beta\left(|\psi(0,0)|_{\mathcal{A}}, t+j\right)+$ $\eta_{2}\left(1 / \epsilon,\left\|\left(u, d_{u}\right)\right\|_{(t, j)}\right)$. However, $\epsilon$ in (15) can be taken arbitrarily large, for which we can always find the corresponding MATI bound. Consequently, $\eta_{2}$ can be made arbitrarily small by reducing MATI so that the effect of $\left(u, d_{u}\right)$ is reduced (i.e. we achieve practical stabilisation). In the absence of inputs, i.e. $w=v=u=0$, the estimation error indeed asymptotically converges to the origin.

${ }^{1}$ See [21] for important definitions related to system (12), in particular,
for a definition of the notion of solution pair. The $\infty$-norm in Theorem
1 is defined, for a hybrid signal $w$ with $(0,0)$ as initial hybrid time,
as $\|w\|_{(t, j)} \doteq \max \left\{\begin{array}{c}\text { ess.sup } \\ \left(t^{\prime}, j^{\prime}\right) \in \operatorname{dom} w \backslash \Gamma(w),(0,0) \preceq\left(t^{\prime}, j^{\prime}\right) \preceq(t, j)\end{array}\right.$ $\left.\sup _{\left(t^{\prime}, j^{\prime}\right) \in \Gamma(w),(0,0) \preceq\left(t^{\prime}, j^{\prime}\right) \preceq(t, j)}\left|w\left(t^{\prime}, j^{\prime}\right)\right|\right\}$, where $\Gamma(w)$ denotes the set of all $\left(t^{\prime}, j^{\prime}\right) \in \operatorname{dom} w$ such that $\left(t^{\prime}, j^{\prime}+1\right) \in \operatorname{dom} w$. 


\section{CASE STUDY}

Consider the non-linear plant

$$
\dot{x}_{p}=A x_{p}+\pi\left(x_{p}\right)+w, \quad y=C x_{p}+v,
$$

where $x_{p} \in \mathbb{R}^{n_{x}}, y \in \mathbb{R}^{n_{y}}, A, B$ and $C$ are constant matrices of appropriate dimensions, and $\pi: \mathbb{R}^{n_{x}} \rightarrow \mathbb{R}^{n_{x}}$ is globally Lipschitz with constant $\Pi \geq 0$. We design the observer

$$
\dot{x}_{o}=A x_{o}+\pi\left(x_{o}\right)+K\left(y-y_{o}\right), \quad y_{o}=C x_{o},
$$

where $x_{o} \in \mathbb{R}^{n_{x}}$ and $K$ is such that the following holds.

Assumption 5: There exist a matrix $K$ and real symmetric positive definite matrix $P$ such that for $V: \chi \mapsto \chi^{T} P \chi$, $\left\langle\nabla V(\chi),(A-K C) \chi+\pi\left(x_{p}+\chi\right)-\pi\left(x_{p}\right)\right\rangle \leq-c V(\chi)$ for all $\chi \in \mathbb{R}^{n_{x}}$ and some $c>a^{2} / \lambda_{\min }(P)$, where $a \doteq$ $(|C(A-K C)|+\Pi|C|)\left(3^{N}-1\right) / 2$.

This assumption is satisfied by high gain observers, see [22] for instance.

The observer (17) is implemented over the WH network, in which transmissions are scheduled by the FDD-RR protocol in Example 1. For this case study,

$$
\begin{aligned}
f_{\chi}(\psi, v, w)= & (A-K C) \chi+\pi\left(x_{p}+\chi\right)-\pi\left(x_{p}\right) \\
& +K\left(\mathbf{1}_{2 \ell_{y}} \cdot \varsigma+v\right)-w \\
f_{p}(\psi, w)= & A x_{p}+\pi\left(x_{p}\right)+w \\
g_{\varsigma}\left(\psi, v, w, d_{v}\right)= & \left(C f_{\chi}(\psi, v, w)-d_{v}, 0, \ldots, 0\right) .
\end{aligned}
$$

We then verify that Assumptions 2-4 hold in the proposition below.

Proposition 1: Consider system (12) with $f_{\chi}, f_{p}$ and $g_{\varsigma}$ as per (18), and $h_{\varsigma}$ and $W$ as per Example 1. Suppose Assumption 5 holds, then

1) Assumption 2 holds with $\underline{\alpha}_{W}=1, \bar{\alpha}_{W}=$ $\sqrt{\left(3^{N}-1\right) / 2}$ and $\lambda=\sqrt{\left(3^{N}-3\right)\left(3^{N}-1\right)}$, where $N=\max \left\{2 \ell_{y}+1,2 \ell_{u}+1\right\}$.

2) Assumption 3 holds with $L=\sqrt{2 \ell_{y}}\left(3^{N}-1\right)|C K| / 2$, $H(\chi)=a|\chi|$, where $a$ comes from Assumption 5, and $\sigma(s)=\left(3^{N}-1\right) \max \{|C K|,|C|, 1\} s / 2$, for all $s \geq 0$.

3) Assumption 4 holds with $V: \chi \mapsto \chi^{T} P \chi, \underline{\alpha}_{V}(s)=$ $\lambda_{\min }(P) s^{2}, \bar{\alpha}_{V}(s)=\lambda_{\max }(P) s^{2}, \varrho=\left(c \lambda_{\min }(P)-\right.$ $\left.a^{2}\right) /\left(1+\lambda_{\min }(P)\right), \gamma=\sqrt{\varrho+(2 / \varrho) \varpi^{2}}$ and $\mu(s)=$ $(4 / \varrho) \varpi^{2} s^{2}$, where $\varpi \doteq \max \left\{\left|P K \cdot \mathbf{1}_{2 \ell_{y}}\right|,|P K|,|P|\right\}$, with $P, c, a>0$ as per Assumption 5 .

A direct consequence of Proposition 1 is that all conditions of Theorem 1 are satisfied. Moreover, all parameters needed to calculate the MATI bound in Theorem 1 are given in Proposition 1. Hence, Theorem 1 can be directly applied.

\section{CONCLUSIONS}

We proposed a Lyapunov-based emulation approach for the observer design of non-linear NCSs over WH. To handle the specific features of $\mathrm{WH}$, we presented a hybrid model for the overall NCS, in which the plant is subject to external disturbances and measurement noise. For an observer designed in the absence of a communication network, we investigated the conditions on the latter to preserve its convergence property. In particular, we provided an explicit MATI bound that ensures that the estimation error verifies an ISS property, provided the scheduling protocols are UGES.

\section{REFERENCES}

[1] J. Hespanha, P. Naghshtabrizi, and Y. Xu, "A survey of recent results in networked control systems," Proceedings of the IEEE, vol. 95, no. 1, pp. 138-162, January 2007.

[2] W. Heemels and N. Van De Wouw, "Stability and stabilization of networked control systems," in Networked Control Systems. Springer, 2010, pp. 203-253.

[3] J. Pesonen, "Stochastic estimation and control over WirelessHART networks: Theory and implementation," Master's thesis, KTH Electrical Engineering, January 2010.

[4] H. Zhang, P. Soldati, and M. Johansson, "Time-and channel-efficient link scheduling for convergecast in WirelessHART networks," in Proceedings of the 13th IEEE International Conference on Communication Technology (ICCT), 2011.

[5] — - "Performance bounds and latency-optimal scheduling for convergecast in WirelessHART networks," IEEE Transactions on Wireless Communications, vol. 12, no. 6, pp. 2688-2696, 2013.

[6] Z. Zou, B. Demirel, and M. Johansson, "Minimum-energy packet forwarding policies for guaranteed LQG performance in wireless control systems," in Proceedings of the 51st Conference on Decision and Control, 2012.

[7] B. Demirel, Z. Zou, P. Soldati, and M. Johansson, "Modular design of jointly optimal controllers and forwarding policies for wireless control," IEEE Transactions on Automatic Control, vol. 59, no. 12, pp. 3252-3265, 2014.

[8] R. Alur, A. d'Innocenzo, K. Johansson, G. Pappas, and G. Weiss, "Compositional modeling and analysis of multi-hop control networks," IEEE Transactions on Automatic control, vol. 56, no. 10, pp. 23452357, 2011.

[9] A. Al-Dabbagh and T. Chen, "A fixed structure topology for wireless networked control systems," in Proceedings of the 55th Conference on Decision and Control, Las Vegas, U.S.A., 2016, pp. 3450-3455.

[10] A. Maass, D. Nešić, and P. Dower, "A hybrid model of networked control systems implemented on WirelessHART networks under source routing configuration," in Australian Control Conference, Newcastle, Australia, 2016.

[11] A. Maass, D. Nešić, R. Postoyan, P. Dower, and V. Varma, "Emulationbased stabilisation of networked control systems over WirelessHART," in Proceedings of the 56th IEEE Conference on Decision and Control, Melbourne, Australia, 2017, pp. 6628-6633.

[12] R. Postoyan and D. Nešić, "A framework for the observer design for networked control systems," IEEE Transactions on Automatic Control, vol. 57, no. 5, pp. 1309-1314, 2012.

[13] R. Postoyan, N. Van de Wouw, D. Nešić, and W. Heemels, "Tracking control for nonlinear networked control systems," IEEE Transactions on Automatic Control, vol. 59, no. 6, pp. 1539-1554, 2014.

[14] W. Wang, D. Nešić, and R. Postoyan, "Observer design for networked control systems with FlexRay," Automatica, vol. 82, pp. 42-48, 2017.

[15] R. Goebel, R. Sanfelice, and A. Teel, Hybrid Dynamical Systems. modeling, stability, and robustness. Princeton University Press, 2012.

[16] D. Chen, M. Nixon, and A. Mok, WirelessHART ${ }^{T M}$ : Real-Time Mesh Network for Industrial Automation. Boston, MA: Springer US, 2010, pp. 195-199.

[17] G. Walsh, O. Beldiman, and L. Bushnell, "Asymptotic behavior of nonlinear networked control systems," IEEE Transactions on Automatic Control, vol. 46, no. 7, pp. 1093-1097, jul 2001.

[18] T. Ahmed-Ali and F. Lamnabhi-Lagarrigue, "High gain observer design for some networked control systems," IEEE Transactions on Automatic Control, vol. 57, no. 4, pp. 995-1000, 2012.

[19] D. Carnevale, A. Teel, and D. Nešić, "A Lyapunov proof of an improved maximum allowable transfer interval for networked control systems," IEEE Transactions on Automatic Control, vol. 52, no. 5, p. 892, 2007.

[20] D. Nešić and A. Teel, "Input-output stability properties of networked control systems," IEEE Transactions on Automatic Control, vol. 49, no. 10, pp. 1650-1667, 2004.

[21] C. Cai and A. R. Teel, "Characterizations of input-to-state stability for hybrid systems," Systems \& Control Letters, vol. 58, no. 1, pp. 47-53, 2009

[22] R. Rajamani, "Observers for lipschitz nonlinear systems," IEEE Transactions on Automatic Control, vol. 43, no. 3, pp. 397-401, Mar 1998. 


\section{University Library}

\section{- M M I N E R VA A gateway to Melbourne's research publications}

Minerva Access is the Institutional Repository of The University of Melbourne

Author/s:

Maass, Al;Nesic, D;Postoyan, R;Dower, PM

Title:

Observer design for networked control systems implemented over WirelessHART

Date:

2018

Citation:

Maass, A. I., Nesic, D., Postoyan, R. \& Dower, P. M. (2018). Observer design for

networked control systems implemented over WirelessHART. Proceedings of the 57th IEEE Conference on Decision and Control (CDC), 2018-December, pp.2836-2841. IEEE. https:// doi.org/10.1109/CDC.2018.8619754.

Persistent Link:

http://hdl.handle.net/11343/251425 